\title{
Amelioration of liver function and glucose control with pioglitazone in a patient with diabetes mellitus type 2 and nonalcoholic fatty liver disease
}

\section{ABSTRACT}

Nonalcoholic fatty liver disease (NAFLD) is the most common cause of hepatic steatosis and elevated aminotransferases in the general population. It is a benign disease; however, in a portion of patients it progresses to nonalcoholic steatohepatitis (NASH) and cirrhosis. The most effective but the most difficult treatment for it is body mass reduction by behavior interventions. Different drugs have been tested in the treatment of NAFLD but their effect was not satisfactory; therefore, there is no regular, causal and pharmacological treatment for NAFLD at the moment. The etiology of NAFLD is unclear but insulin resistance is believed to play a significant pathogenic role. Data concerning the possibilities of using oral antidiabetic drugs in the treatment of NAFLD are limited. Among insulin sensitizers, metformin is a first-line drug in the treatment of diabetes type 2; however, due to its gastro-intestinal adverse reactions, some patients cannot use it or cannot achieve an optimal treatment dose. On the other hand, the drug is contraindicated for some patients. In this case study, we present a patient with diabetes mellitus type 2 with elevated aminotransferases levels

Address for correspondence:

dr n. med. Katarzyna Nabrdalik

Katedra i Klinika Chorób Wewnętrznych, Diabetologii i Nefrologii

Wydział Lekarski z Oddziałem Lekarsko-Dentystycznym w Zabrzu,

Śląski Uniwersytet Medyczny w Katowicach

e-mail: knabrdalik@yahoo.com

Translation: GROY Translations

Clinical Diabetology 2016, 5, 6, 199-202

DOI: $10.5603 /$ DK.2016.0034

Received: 12.01 .2017

Accepted: 09.03.2017 related to NAFLD and inadequate glucose control, who has been treated with a sulphonylurea derivative and dipeptydylo peptidase IV inhibitor. Replacement of previously used drugs by basal insulin and pioglitazone resulted in both the reduction of the activity of liver enzymes and the improvement in glucose control. (Clin Diabetol 2016; 5, 6: 199-202)

Key words: diabetes type 2, NAFLD, NASH, pioglitazone

\section{Introduction}

Nonalcoholic fatty liver disease (NAFLD), which is defined as hepatic steatosis without the presence of secondary causes (such as genetic factors, alcohol, drugs), is currently the most common cause of chronic liver disease in many developed countries, and its incidence rate is constantly increasing worldwide. In order to assess the severity of the disease and its progression risk to cirrhosis, NAFLD is divided into two categories: nonalcoholic fatty liver (NAFL) and nonalcoholic steatohepatitis (NASH). The difference between both of these medical conditions is histological [1]. One third of the world population display characteristics of hepatic steatosis in imaging examinations and most of the population (70-90\%) demonstrate signs of NAFLD. $\mathrm{NASH}$, which is a progressive disease, coexists with around $10-30 \%$ of the people affected by NAFLD. On the other hand, cirrhosis, along with the risk of hepatocellular carcinoma development, may be developed in even one third of the patients with NASH. Currently, 
NASH is the second most common cause for liver transplantation and it is projected that it will become the main cause by 2020 [2].

In most patients, the course of NAFLD is asymptomatic and it is diagnosed coincidentally: usually when abnormal laboratory results are presented concerning the activity of liver enzymes, especially aminotransferases, or features of hepatic steatosis in an imaging examination. It should be noted that not all NAFLD cases exhibit elevated liver enzymes activity, and the activity level does not correspond to the degree of hepatitis or cirrhosis [3].

NAFLD diagnosis requires the confirmation of hepatic steatosis features in an imaging examination and/ /or in a histological examination and the elimination of other causes of hepatic steatosis. The gold standard of the diagnosis of NAFLD is a liver biopsy but it should be reserved for diagnostically doubtful cases [4].

NAFLD affects nearly $70 \%$ of patients suffering from diabetes type 2 and diabetes per se is considered as a risk factor for NAFLD and its progression to more advanced phases of the disease, including cirrhosis and hepatocellular carcinoma [5]. Furthermore, patients with NAFLD have a higher risk of cardiovascular diseases and cardiovascular mortality [6].

The pathogenesis of NAFLD is unclear but it probably involves multiple processes and takes into account genetic, metabolic and inflammatory factors, poor diet and dysfunctions concerning the gut flora [7-9]. However, it is insulin resistance that is believed to be the main contributing factor. It initiates the fat storage in the liver, oxidative stress and lipotoxicity [10]. It is worth noting that insulin resistance contributes to the development of obesity, diabetes type 2 and NAFLD, even in people whose body mass index (BMI) is within the normal range, which has been observed in tests using the euglicemic clamp technique [11].

The guidelines concerning the treatment for NAFLD, which refer to the general population, may also be used in patients with diabetes type 2. A single, but the most important and the most difficult, therapeutic recommendation in treating NAFLD is to achieve and maintain the normal body mass. Any attempts to determine targeted pharmacological treatment have been unsuccessful so far [12]. Pioglitazone, included in the thiazolidinedione class, seems to be a promising drug to treat NAFLD. Due to its antagonistic effect on peroxisome proliferator-activated receptors gamma (PPAR gamma), it has a positive impact on glucose regulation and lipid metabolism. The results of the studies that have been conducted so far indicate that pioglitazone treatment leads to the reduction of hepatitis and cirrhosis features [13, 14]. Performed studies which related to the effectiveness of pioglitazone in NAFLD treatment, have concerned the assessment of hepatitis and cirrhosis only: without the evaluation of the amelioration of glucose control. In this case study, we present a patient with diabetes type 2 and NAFLD, who has significantly improved glucose control and reduced elevated aminotransferases activity as a result of pioglitazone treatment.

\section{Case study}

In June 2016, the 54-year-old patient, who has suffered from diabetes type 2, hypertension and dyslipidaemia for 10 years, has been admitted to the Diabetology Ward of the Department of Internal Medicine, Diabetology and Nephrology in Zabrze due to poor diabetes control (mean blood glucose levels in self control $220 \mathrm{mg} / \mathrm{dL}$ while fasting and $250 \mathrm{mg} / \mathrm{dL}$ 2 hours after a meal). In 2014, the patient was diagnosed with NAFLD based on a liver biopsy. The medicines taken in the diabetes treatment included a sulphonylurea derivative (glimepiride $4 \mathrm{mg} / \mathrm{d}$ ) and dipeptydylo peptidase IV inhibitor (linagliptin $5 \mathrm{mg} / \mathrm{d}$ ). In addition, the patient took an angiotensin-converting-enzyme inhibitor (ramipril $10 \mathrm{mg}$ in the evening) due to concomitant hypertension with a good antihypertensive effect. Despite concomitant dyslipidaemia, the patient did not take statins due to aminotransferases activity 3 times higher than the reference values. The patient attempted only to follow a diet with limited animal fat. Metformin was not used in the therapy due to the adverse reactions that this medicine had caused in the past. The family history was positive in regard of diabetes type 2 and cardiovascular deaths. Features of hepatic steatosis in abdominal ultrasound imaging as well as 4-5 times higher aminotransferases activity than the reference values had been observed for approx. 3 years. Consequently, the patient visited a hepatology clinic and had a liver biopsy, which diagnosed NAFLD. After the patient was admitted to the ward, no abnormalities were found in the physical examination; $\mathrm{BMI}=24 \mathrm{~kg} / \mathrm{m}^{2}$. The mean arterial blood pressure, measured three times in a roll in a sitting position, was $140 / 80 \mathrm{~mm} \mathrm{Hg}$. In laboratory tests: $\mathrm{HbA}_{1 \mathrm{c}}=9 \%$ $(75 \mathrm{mmol} / \mathrm{mol})$, ALAT $=164 \mathrm{U} / \mathrm{L}$, ASPAT $=137 \mathrm{U} / \mathrm{L} . \mathrm{GGTP}$ - within the reference values, HBsAg negative, antibodies (anti-HCV) negative, serum creatinine, eGFR, the results of the tests of morphotic elements of the blood and general urinalysis within the reference values. Total cholesterol was $220 \mathrm{mg} / \mathrm{dL}$, LDL cholesterol $148 \mathrm{mg} / \mathrm{dL}$, $\mathrm{HDL}$ cholesterol $42 \mathrm{mg} / \mathrm{dL}$ and triglycerides - $152 \mathrm{mg} / \mathrm{dL}$. Abdominal ultrasound imaging confirmed the presence of the hepatic steatosis features. Ophthalmoscopy showed no signs of diabetic retinopathy. Due to 
inadequate blood glucose levels control following the current scheme of combination therapy, the patient was advice to stopped taking the sulphonylurea derivative and DPP4 inhibitor and to begin treatment with basal insulin (12 units of NPH insulin) taken before going to bed at night in combination with pioglitazone at a daily dose of $30 \mathrm{mg}$. During the hospitalization, spectacular results were already achieved both in blood glucose levels while fasting (mean blood glucose levels $130 \mathrm{mg}$ / $/ \mathrm{dL}$ ) as well as postprandial (140 mg/dL). The patient was discharged from the Ward with the recommendation to follow the treatment in outpatient clinics under the supervision of a Diabetes Clinic and Hepatology Clinic. The patients visited the Diabetes Clinic one month after the discharge. A further and significant amelioration of blood glucose control was observed. Self-controlled mean blood glucose levels while fasting were $110 \mathrm{mg} / \mathrm{dL}$ and postprandial $120 \mathrm{mg} / \mathrm{dL}$, without hypoglycaemia episodes. Laboratory tests revealed the reduction of liver enzymes activity: ALAT $=92 \mathrm{U} / \mathrm{L}$ and ASPAT $=75 \mathrm{U} / \mathrm{L}$. Due to dyslipidaemia and a high cardiovascular risk, it was decided to add to the treatment atorvastatin in $20 \mathrm{mg} / \mathrm{d}$ dose and the patient was also recommended to continue the treatment with pioglitazone in combination with basal insulin at the same doses. The patient visited the Diabetes Clinic after another 3 months. $\mathrm{HbA}_{1 \mathrm{c}}$ value was $7 \%$ and further reductions in the activity of liver enzymes was noticed (ALAT $=64 \mathrm{U} / \mathrm{L}$ and ASPAT $=40 \mathrm{U} / \mathrm{L}$ ), as well as the improvement in the lipid profile (total cholesterol - $165 \mathrm{mg} / \mathrm{dL}$, LDL cholesterol - $97 \mathrm{mg} / \mathrm{dL}$; HDL cholesterol - $40 \mathrm{mg} / \mathrm{dL}$, triglycerides - $140 \mathrm{mg} / \mathrm{dL}$ ). The patient was recommended to continue the current treatment.

\section{Conclusions}

The case study of the patient with diabetes type 2 and NAFLD confirms the possibility of treating patients whose $\mathrm{HbA}_{1 \mathrm{c}}$ values are not within the target range and who have significantly elevated aminotransferases activity with pioglitazone effectively. Treatment with pioglitazone is especially effective when the dominant cause of poor diabetes control is insulin resistance. It is worth to remember that normal BMI does not exclude the presence of excessive abdominal fat; therefore, both NAFLD and insulin resistance may affect people who are not overweight or obese. In view of the fact that NAFLD is the most common cause of abnormalities in laboratory and imaging tests of the liver, it is important to popularize knowledge about the disease and its treatment possibilities. Furthermore, it should be kept in mind that even though hepatic steatosis is asymptomatic, epidemiological data indicate a higher incidence of cirrhosis and hepatocellular carcinoma, as well as cardiovascular complications, in this group of patients. Studies that evaluate the effect of pharmacological treatment for NAFLD are very diverse depending on the criteria for the qualification for a study (nonalcoholic fatty liver through NASH and cirrhosis), as well as on primary endpoints. There are no registered medications for causal treatment of NAFLD at the moment. The most important therapeutic recommendation is behavioral intervention, which involves the proper diet and regular physical activity, both of which are beneficial for people with NAFLD and have a positive impact on cardiovascular risk factors $[15,16]$. Furthermore, it should be remembered that patients with NAFLD, diabetes and dyslipidaemia are high cardiovascular risk patients and statin treatment should be considered for them. Especially that in accordance with the opinion of the experts in liver disease treatment (Liver Expert Panel) from 2006, it is emphasized that such treatment is safe, and a therapy targeted at the reduction of the risk of cardiovascular complications is more important than the potential risk of hepatotoxicity associated with statin treatment [17].

\section{REFERENCES}

1. Milic S, Stimac D. Nonalcoholic fatty liver disease/steatohepatitis: epidemiology, pathogenesis, clinical presentation and treatment. Digestive diseases (Basel, Switzerland). 2012; 30: 158-162.

2. Wree A, Broderick L, Canbay A, Hoffman HM, Feldstein AE. From NAFLD to NASH to cirrhosis-new insights into disease mechanisms. Nat Rev Gastroenterol Hepatol 2013; 10: 627-636.

3. Mofrad P, Contos MJ, Haque M et al. Clinical and histologic spectrum of nonalcoholic fatty liver disease associated with normal ALT values. Hepatology (Baltimore, Md) 2003; 37: 1286-1292.

4. Chalasani N, Younossi Z, Lavine JE et al. The diagnosis and management of non-alcoholic fatty liver disease: practice Guideline by the American Association for the Study of Liver Diseases, American College of Gastroenterology, and the American Gastroenterological Association. Hepatology (Baltimore, Md) 2012; 55: 2005-2023.

5. El-Serag HB, Tran T, Everhart JE. Diabetes increases the risk of chronic liver disease and hepatocellular carcinoma. Gastroenterology 2004; 126: 460-468.

6. Targher G, Bertolini L, Padovani R et al. Prevalence of nonalcoholic fatty liver disease and its association with cardiovascular disease among type 2 diabetic patients. Diabetes Care 2007; 30: 1212-1218.

7. Kozlitina J, Smagris E, Stender S et al. Exome-wide association study identifies a TM6SF2 variant that confers susceptibility to nonalcoholic fatty liver disease. Nature Genetics 2014; 46: 52-56.

8. Vos MB, Lavine JE. Dietary fructose in nonalcoholic fatty liver disease. Hepatology (Baltimore, Md) 2013; 57: 2525-2531.

9. Mehal WZ. The Gordian Knot of dysbiosis, obesity and NAFLD. Nature reviews Gastroenterology \& Hepatology 2013; 10: 637-644.

10. Birkenfeld AL, Shulman GI. Nonalcoholic fatty liver disease, hepatic insulin resistance, and type 2 diabetes. Hepatology (Baltimore, Md) 2014; 59: 713-723

11. Bugianesi E, Gastaldelli A, Vanni E et al. Insulin resistance in non-diabetic patients with non-alcoholic fatty liver disease: sites and mechanisms. Diabetologia 2005; 48: 634-642.

12. Malinowski SS, Byrd JS, Bell AM, Wofford MR, Riche DM. Pharmacologic therapy for nonalcoholic fatty liver disease in adults. Pharmacotherapy 2013; 33: 223-242. 
13. Cusi K. Treatment of patients with type 2 diabetes and nonalcoholic fatty liver disease: current approaches and future directions. Diabetologia 2016; 59: 1112-1120.

14. Rakoski MO, Singal AG, Rogers MA, Conjeevaram H. Metaanalysis: insulin sensitizers for the treatment of non-alcoholic steatohepatitis. Alimentary Pharmacology \& Therapeutics 2010; 32: 1211-1221.

15. Choudhary NS, Saraf N, Saigal S et al. Rapid reversal of liver steatosis with life style modification in highly motivated liver donors. Journal of Clinical and Experimental Hepatology 2015; 5: 123-126.

16. Hallsworth K, Thoma C, Hollingsworth KG et al. Modified high-intensity interval training reduces liver fat and improves cardiac function in non-alcoholic fatty liver disease: a randomized controlled trial. Clinical Science (London) 2015; 129: 1097-1105 .

17. Cohen DE, Anania FA, Chalasani N. An assessment of statin safety by hepatologists. Am J Cardiol 2006; 97: 77c-81c. 